\title{
Hippocampal oscillations in the rodent model of schizophrenia induced by amygdala GABA receptor blockade
}

\section{Tope Lanre-Amos and Bernat Kocsis*}

Department of Psychiatry, Beth Israel Deaconess Medical Center, Harvard Medical School, Boston, MA, USA

\section{Edited by:}

Miles A. Whittington,

Newcastle University, UK

Reviewed by:

Miles A. Whittington,

Newcastle University, UK

Andre Fisahn,

Karolinska Institute, Sweden

*Correspondence:

Bernat Kocsis,

Department of Psychiatry, Beth Israel

Deaconess Medical Center,

Harvard Medical School, 330 Brookline

Ave, Boston, MA 02215, USA.

e-mail:bkocsis@hms.harvard.edu
Brain oscillations are critical for cognitive processes, and their alterations in schizophrenia have been proposed to contribute to cognitive impairments. Network oscillations rely upon GABAergic interneurons, which also show characteristic changes in schizophrenia. The aim of this study was to examine the capability of hippocampal networks to generate oscillations in a rat model previously shown to reproduce the stereotypic structural alterations of the hippocampal interneuron circuit seen in schizophrenic patients. This model uses injection of GABA-A receptor antagonist picrotoxin into the baso-lateral amygdala which causes cell-type specific disruption of interneuron signaling in the hippocampus. We found that after such treatment, hippocampal theta rhythm was still present during REM sleep, locomotion, and exploration of novel environment and could be elicited under urethane anesthesia. Subtle changes in theta and gamma parameters were observed in both preparations; specifically in the stimulus intensity theta frequency relationship under urethane and in divergent reactions of oscillations at the two major theta dipoles in freely moving rats. Thus, theta power in the CA1 region was generally enhanced as compared with deep theta dipole which decreased or did not change. The results indicate that pathologic reorganization of interneurons that follows the over-activation of the amygdala-hippocampal pathway, as shown for this model of schizophrenia, does not lead to destruction of the oscillatory circuit but changes the normal balance of rhythmic activity in its various compartments.

Keywords: theta rhythm, gamma rhythm, hippocampal interneurons, theta dipoles, REM sleep, novelty exploration, parvalbumin, cholecystokinin

\section{INTRODUCTION}

Schizophrenia, a neurodevelopmental disorder, is marked by abnormalities in sensory processing and cognition. Neural oscillations and synchrony are critical for these functions and their disruptions may play an important role in the pathophysiology of schizophrenia. Deficiencies in oscillation power of schizophrenics, as compared to controls, have been demonstrated during sensory processing as well as various cognitive tasks (Krishnan et al., 2005; Schmiedt et al., 2005; Basar-Eroglu et al., 2007; Spencer et al., 2008b; Williams et al., 2009). These abnormalities are also present in first-degree relatives of schizophrenics (Hong et al., 2004) and in first-episode schizophrenia (Spencer et al., 2008a) suggesting that they might be heritable and are not induced by medication (Gallinat et al., 2004).

Although recent research is primarily focusing on high frequency oscillations, there is also evidence of disturbances in slow rhythms in the delta and theta bands in schizophrenia (Koenig et al., 2001; Ford et al., 2002; Schmiedt et al., 2005; Basar-Eroglu et al., 2008; Bates et al., 2009). Functional deficits are most likely the consequence of parallel impairment of these processes as oscillations at different frequencies have overlapping neuronal substrates (Buzsaki et al., 1992; Klausberger and Somogyi, 2008) and are hierarchically organized such that slow rhythms drive coordinated shifting of excitability in local neuronal ensembles and optimize gamma dynamics (Lakatos et al., 2008; Lisman and Buzsaki, 2008). GABAergic interneurons are critical for both gamma and theta oscillations and their deficiencies are well documented in schizophrenic patients as well as in animal models of schizophrenia. Of the numerous classes of GABAergic cells, parvalbumin expressing $(\mathrm{PV}+)$ interneurons gained specific attention. Along with a decrease in GAD expression and a compensatory increase in GABA receptor binding, the loss of $\mathrm{PV}+$ cells is the most common histological hallmark in postmortem investigations in humans (Beasley and Reynolds, 1997; Zhang and Reynolds, 2002) and was described in different animal models including pharmacologic (Keilhoff et al., 2004; Rujescu et al., 2006), neurodevelopmental (Penschuck et al., 2006; Tseng et al., 2008; Francois et al., 2009; Lodge et al., 2009) and genetic (Hikida et al., 2007; Jaaro-Peled et al., 2010; Steullet et al., 2010). Less data are available about other types of interneurons which may also be involved, although they are unlikely to be as critical as the fast firing, non-accommodating PV+ cells. Thus, the most widely accepted hypothesis identifies PV+ cell pathology as the direct cause of impaired oscillations. Electrophysiological examination, however, of the effect of structural changes on oscillations has only started in the past several years, and only in a few of these models (Lodge et al., 2009; Lazarewicz et al., 2010; Vohs et al., 2010).

The goal of this study was to examine the functional capacity of the oscillatory circuits of the hippocampus in one of the rodent models of schizophrenia which had previously been shown to reproduce the stereotypic structural damage of the hippocampus seen in schizophrenic patients (Berretta et al., 2001, 2004; 
Berretta and Benes, 2006). This model uses injection of picrotoxin, a non-competitive GABA-A receptor antagonist, into the basolateral amygdala (BLA), which strongly projects to the hippocampus (Pikkarainen et al., 1999) and releases glutamate to drive the interneurons of this region. Disinhibition of BLA neurons causes delayed excitotoxicity in the hippocampus to which the PV+ interneurons are uniquely sensitive. Face validity of this partial model is based on a pattern of changes in interneurons which mimic postmortem findings in human schizophrenics. Importantly, extensive quantitative histological data is available for this model which reveals not only PV+ cell loss, described in most models, but also various other aspects of reorganization of the network, including other classes of interneurons. i.e., expressing cholecystokinin (CCK), or calretinin (CR), and their regional distribution from the dentate gyrus (DG) through sections CA1-4 of Ammon's horn (Berretta et al., 2001, 2004). Furthermore, in addition to cell numbers, $\mathrm{PV}+$, and CCK+ terminals were also counted across different regions, and the correlation between these markers was quantitatively analyzed. It was shown in particular, that expression of such markers in hippocampal interneurons is under the control of interneuronal networks encompassing multiple hippocampal sectors and that perturbation of amygdalar input triggered a chain of long-term changes involving multiple interconnected subpopulations of interneurons (Berretta et al., 2004). However, as was argued regarding structural alterations in neuronal networks in schizophrenia (Lewis et al., 2005; Gonzalez-Burgos and Lewis, 2008) not all changes necessarily represent successive steps of decline but some may be compensatory and may thus exert complex sum effects on oscillations. In this study, we studied chronic changes in theta and gamma oscillations using local field potential recordings in the hippocampus at critical time points after BLA picrotoxin injection, primarily focusing on theta rhythm associated with different behaviors in freely behaving rats or elicited by brainstem stimulation under urethane anesthesia.

\section{MATERIALS AND METHODS SURGERY, ELECTRODE IMPLANTS}

All experiments followed the recommendations of the Guide for the Care and Use of Laboratory Animals and were approved by the Institutional Animal Care and Use Committee of the Beth Israel Deaconess Medical Center. Survival surgeries were performed under deep anesthesia using a mixture of ketamine and xylazine $(70-90 \mathrm{mg} /$ $\mathrm{kg}$ b.w. initial dose, supplemented every 35-40 min as necessary). After surgery, the rats were given analgesic (Buprenorphine, $0.05 \mathrm{mg} /$ kg b.w. subcutaneously) and local antibacterial treatment (Bacitracin ointment) and were monitored for 2 days. For field potential recordings, pairs of 125-um stainless steel wires with $\sim 1$-mm tip separation were implanted in the hippocampus on both sides. One of the electrodes were positioned at or below the hippocampal fissure (AP:-3.7, L:2.2, DV:-3.5 mm relative to bregma), and the other in or above the CA1 pyramidal layer in order to pick up signals from the two major theta dipoles. Hippocampal EEG was recorded either under urethane anesthesia in a bipolar electrode arrangement to maximize theta amplitude or in freely moving condition using monopolar recordings to simultaneously monitor activity in the two layers with a common reference over the cerebellum. Theta oscillations in these locations are in opposite phase to each other which allows verification of the electrode placement during recordings. The animals were grounded through a screw electrode implanted in the nasal bone. Rats recorded under urethane were also implanted with electrodes in the pontine reticular formation (RPO, AP:-7.8, L:-1.8 mm, DV:8.0 mm; Figure 1A) to generate evoked theta rhythm by electrical RPO stimulation ( $5 \mathrm{~s}$ trains of $0.2 \mathrm{~ms}$ square waves at $100 \mathrm{~Hz}$; intensity varied between subthreshold and supramaximal in terms of eliciting theta of different frequencies - see Figure 3) (Kocsis and Li, 2004; Siok et al., 2006). In freely moving rats, cortical EEG over the frontal cortex (skull screw, AP:+1.0, L:1.0) and neck muscle EMG were also recorded to determine sleep-wake states. All electrodes were fixed to the skull with dental acrylic and connected to a miniature connector.

\section{MICROINJECTION OF PICROTOXIN IN THE BLA}

Microinjection of picrotoxin in the BLA followed three different protocols in separate experiments. In all rats, picrotoxin $(62.5 \mathrm{ng} / \mathrm{ml}$ dissolved in $0.9 \%$ saline) was injected $(80 \mu$ lvolume, rate: $20 \mu \mathrm{l} / \mathrm{min})$ in the BLA in (AP: $-2.5 \mathrm{~mm}, \mathrm{ML}:-4.8 \mathrm{~mm}, \mathrm{DV}: 8.2$ to bregma), as described in (Berretta and Benes, 2006). In two protocols, injection was performed under anesthesia using a Hamilton syringe $(100 \mu \mathrm{l})$ lowered slowly and kept in place for 20 min before injection. In the first protocol (acute experiment), recordings started under urethane anesthesia $1 \mathrm{~h}$ before and lasted 90 after injection. In the second (subchronic), the injection was made under ketamine and xylazine anesthesia and the electrode implants and recordings were made 4 days later under urethane. In the third protocol, in chronic experiments, $22 \mathrm{G}$ cannulas were inserted $0.2 \mathrm{~mm}$ above the BLA on both sides, during the same surgery as the electrode implants (Figure 1B). In these rats, recordings started 7-10 days after surgery and picrotoxin was injected 7 days later on one side and after another 8 or 9 days on the other side. A group of rats also received saline injection on one side, 1 week before picrotoxin, through the same cannula. At the end of each experiment, the animals were perfused and the location of the cannulas were verified in brain slices stained with cresyl violet.

\section{ELECTROPHYSIOLOGICAL RECORDINGS}

Electrophysiological recordings in chronic experiments were made on days 2, 4, and 8 after each injection. For recordings, the rats were placed in their individual recording boxes and kept connected to the recording apparatus undisturbed, for $3 \mathrm{~h}$ during the light part of the day. Three days before the first injection and on day 4 after each injection, the recording session ended with a 15 min recording in a novel environment (NOV), in a large box with unfamiliar objects.

\section{ELECTROPHYSIOLOGICAL SIGNAL PROCESSING}

EEG signals were amplified, filtered and subjected to analog-todigital conversion. The filter setting, and the conversion rate were different for recordings under anesthesia and in freely moving condition; $1-70 \mathrm{~Hz}$ vs. $0.3-100 \mathrm{~Hz}$, and $1 \mathrm{kHz}$ vs. $250 \mathrm{~Hz}$, respectively. Power spectra $(0.25 \mathrm{~Hz}$ resolution) were generated using the Fast Fourier Transform on 4-s windows. In recordings under anesthesia, power spectra were calculated separately for segments during and outside of RPO stimulation. In chronic experiments, the power spectra were averaged over the 1st hour and hours 2-3 
of the recording sessions (see in Results) and then separately over theta-intensive segments identified as rapid eye movement (REM) sleep (EMG atonia associated with hippocampal theta/delta ratio above 10) and waking exploration (hippocampal theta associated with high EMG activity), using the same recordings as well as over the 15 min period in the novelty box. Theta power was quantified using peak amplitude and frequency of the average spectra as well as average power in the 4-9 and $5-10 \mathrm{~Hz}$ range, in anesthetized and unanesthetized rats, respectively. Gamma power was calculated in chronic experiments as average power in the $30-50 \mathrm{~Hz}$ range.

\section{STATISTICAL ANALYSIS}

In the acute experiment, the changes in spontaneous and RPOelicited theta power, averaged over consecutive 30-min segments, were tested by one-way ANOVA with post hoc comparison of pre-and post-injection segments using one-sided Dunnett test. Differences between control and treatment groups were tested using $t$-test. In subchronic experiments, the slopes of the stimulus intensity-theta frequency relationship were calculated for each rat and group averages were compared between BLA injected rats and anatomical controls using $t$-test. The effect of the injection in chronic experiments was tested using two-way ANOVA with time (three recordings pre and four post-injection) and area (CA1 and DG) as main factors with post hoc comparisons using Bonferroni test.

\section{RESULTS}

As histological alterations resembling those in schizophrenic patients were reported to occur in this model 4 days after picrotoxin injection into the BLA (Berretta et al., 2004; Berretta and Benes, 2006), our investigation focused on the possible disturbances in hippocampal oscillations that could arise at this time point. Spectral analysis of hippocampal field potentials of picrotoxin injected rats was performed in two experiments. The first investigated the functional capacity of the hippocampal network to generate theta rhythm in response to excitatory input from the brainstem reticular formation under urethane anesthesia. The second examined whether theta and gamma oscillations of this circuit had been compromised in freely moving rats during natural theta-intensive behaviors of REM sleep and exploration of a NOV. In addition to the critical 96-h time point, we also tested the acute effect of picrotoxin injection into the BLA on spontaneous and brainstem-elicited hippocampal theta rhythm under anesthesia and its chronic effect on the hippocampal EEG in freely moving rats regularly monitored for 7 days before and 10 days after injection.

\section{ACUTE INCREASE IN HIPPOCAMPAL THETA POWER AFTER PICROTOXIN INJECTION IN BLA}

The acute effect of pharmacological disinhibition of the amygdala-hippocampal pathway was tested in a pilot study on four rats (see example in Figure 2). In control recordings, the hippocampal activity alternated between an irregular pattern and rhythmic slow waves in the low theta frequency range $(3-5 \mathrm{~Hz})$. RPO stimulation elicited regular high frequency $(\sim 7-8 \mathrm{~Hz})$ theta oscillations. After picrotoxin injection in the BLA the hippocampal EEG was dominated by spontaneous theta activity of increased amplitude (see $5 \mathrm{~Hz}$ peak in the power spectrum, Figure 2D "spont"). The amplitude of elicited theta also increased whereas its frequency changed slightly to produce a sharp peak at $8 \mathrm{~Hz}$ in the power spectrum. The changes in hippocampal activity of the rat in Figure 2 developed progressively, reaching a plato within 20-30 min, and lasted for the entire length of the experiment ( $>90 \mathrm{~min}$ ) (Figure 2B). The effect was statistically evaluated using the spectral power
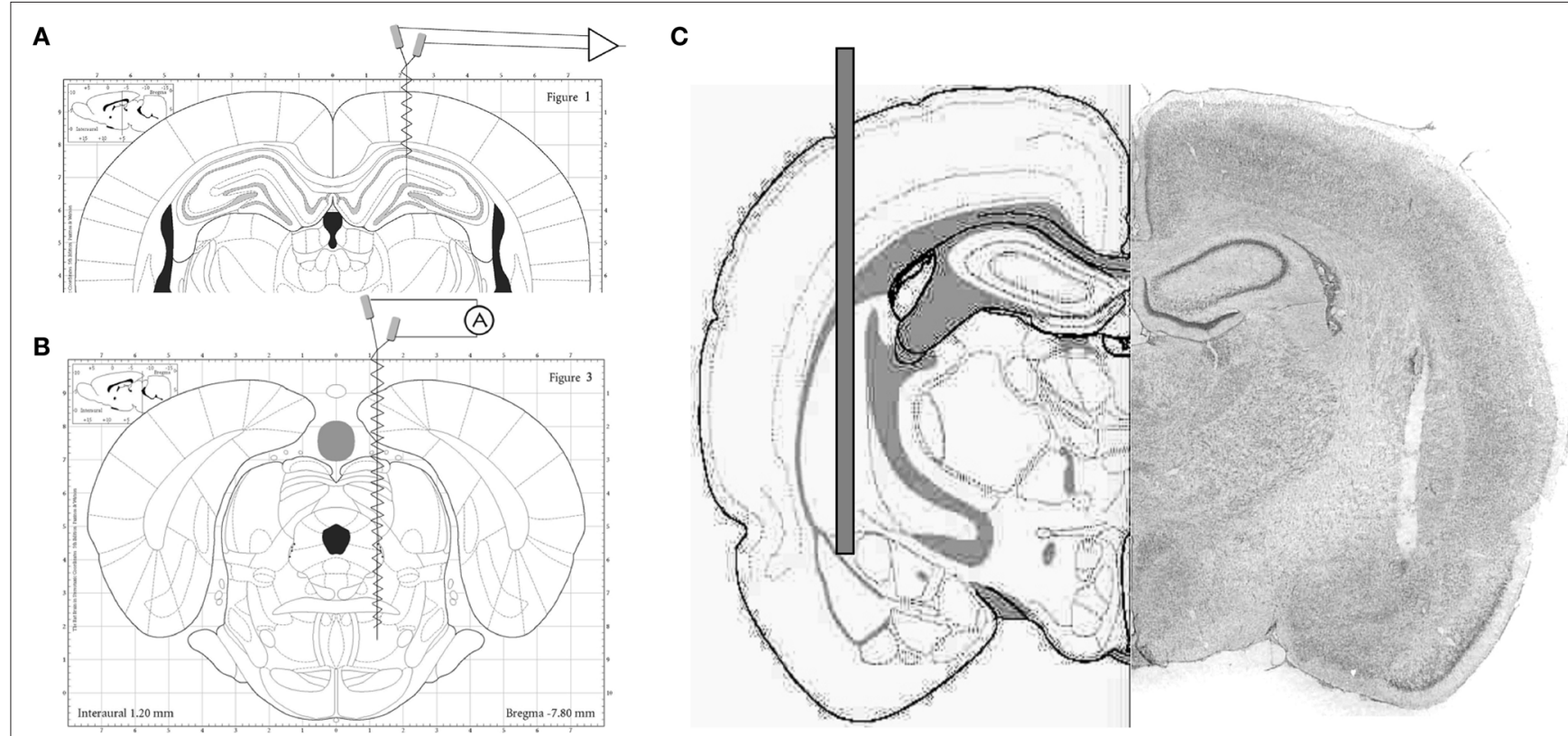

FIGURE 1 | (A) Stereotaxic diagram of electrode placement for hippocampal EEG recordings. (B) Stereotaxic diagram of electrode placement for electrical stimulation of the pontine reticular formation. (C) Stereotaxic diagram (left) and histological reconstruction of the injection cannula targeting the baso-lateral amygdala. (Schematic drawings are from stereotaxic atlases, (A, B) Paxinos, (C) Pellegrino). 


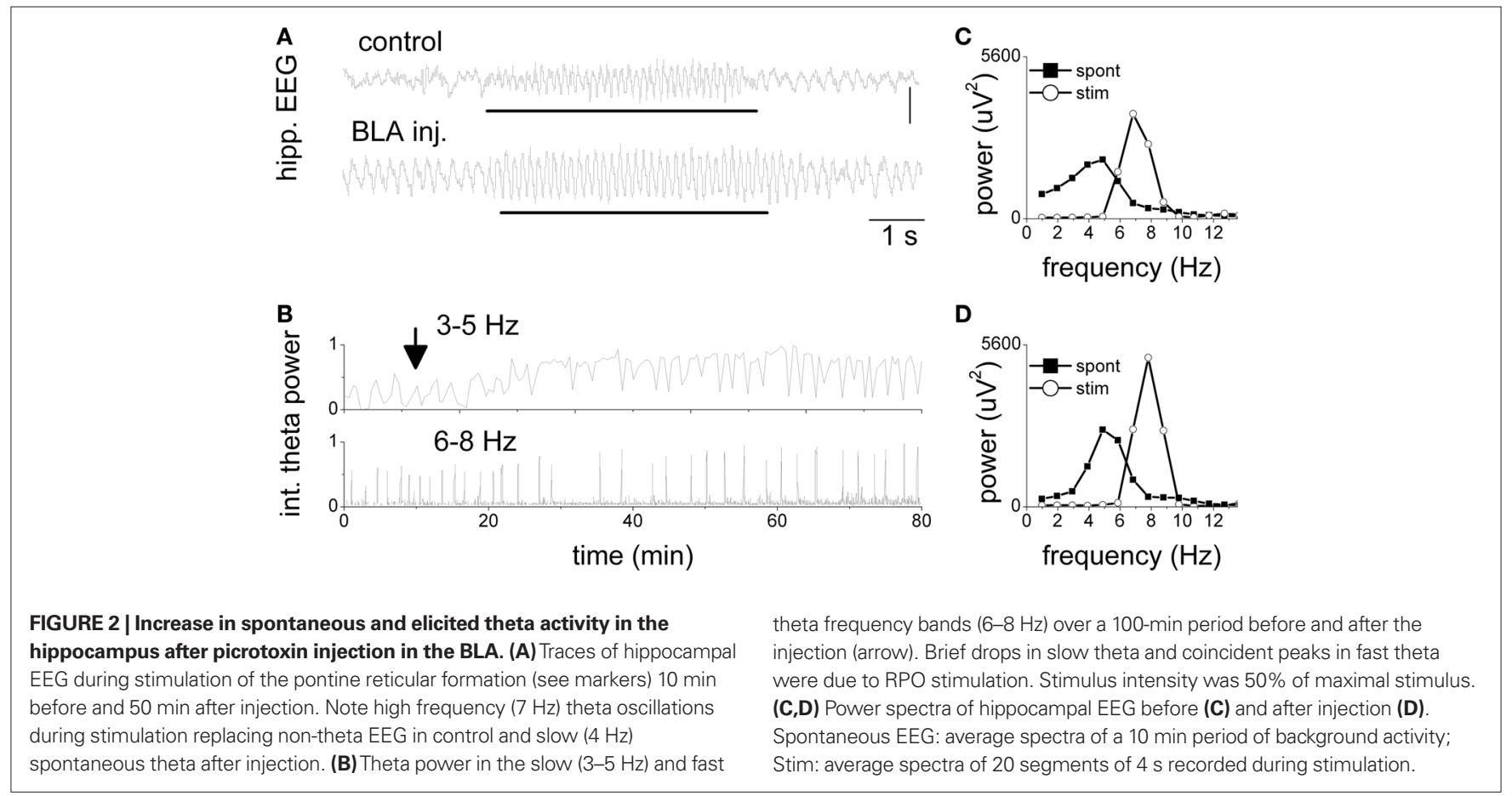

calculated in the $6-8 \mathrm{~Hz}$ range for the segments of elicited theta and was found significantly different between episodes before and after injection ( $p$-values $<0.01$, one-way ANOVA, Dunnett post hoc $t>$ control) and between BLA injection and anatomical controls $(t[85,7]=10.46, p<0.001, t$-test, two-tailed $)$.

\section{DELAYED ALTERATION IN HIPPOCAMPAL THETA RHYTHM AFTER PICROTOXIN INJECTION IN BLA IN URETHANE ANESTHETIZED RATS}

In the next experiment, we recorded hippocampal EEG 4 days ( $96 \mathrm{~h}$ ) after injection of picrotoxin in the amygdala, following the protocol of (Berretta and Benes, 2006). The recordings were made under urethane anesthesia, which unlike other anesthetics does not eliminate theta rhythm and allows exclusion of behavioral effects on theta generation. Since however, urethane can only be used in terminal experiments (i.e., no control recording before injection) the statistical analysis in these experiments was based on comparisons between treatment and control groups using a standard assay based on the known robust linear correlation between theta frequency and the intensity of electrical stimuli applied to RPO (Kocsis and Li, 2004; Siok et al., 2006). Theta rhythm was elicited by RPO stimulation at different intensities in 10 rats $(6 \mathrm{BLA}$ injected and 4 anatomical controls). The effective range of stimulus intensities was individually determined for each rat and the stimulus intensity vs. theta frequency relationship was quantified by the slope of linear regression within this range. Progressive increase in the stimulation intensity from threshold to maximal stimuli produced an upward shift in theta frequency following a linear trend, in both the control and treatment groups (Figures 3A,B). Statistical comparison of the rate of increase in peak theta frequency showed, however, that BLA injected rats were significantly less sensitive to increases in stimulation intensity (Figure 3C). The slope of the control group was $10.4 \pm 1.4 \mathrm{~Hz} / \mathrm{mA}$. The slope of the treatment group showed higher interindividual variability; in three out of six treatment rats it fell within the normal range, although below the control average, whereas in the other three rats the slope was more than 1.7 SD below the average of the control group. The group average for the BLA injected rats was $6.5 \pm 1.1 \mathrm{~Hz} / \mathrm{mA}$ yielding a significant difference between BLAtreated and control groups $(t[6]=2.34, p=0.029, t$-test, 2 sided $)$. As in the acute experiments, there was no difference between left and right hemispheres even though the injections were one-sided $(t[254]=0.37, p=0.35$, paired $t$-test, 2 -tailed $)$.

\section{LACK OF GROSS ALTERATIONS IN HIPPOCAMPAL EEG AFTER PICROTOXIN INJECTION IN BLA IN FREELY MOVING RATS}

In chronic experiments, hippocampal field potentials were recorded in different layers using monopolar recordings. Thus, special care had to be taken to only include rats with precise electrode placement, i.e., in which theta in the two electrodes appeared at $\sim 180^{\circ}$ phase relative to each other, indicating that both the superficial (CA1) and deep theta dipoles (DG) were recorded simultaneously (Buzsaki et al., 1986) (Figure 4B). The rats received picrotoxin injection in the BLA $(n=5)$ or in an area medial to the BLA ( $n=2$, anatomical control) on the right side, followed by another injection on the opposite side, 8-9 days later. Hippocampal EEG was recorded 3 or 4 times during a 7-day period before the first injection, as well as on days 2,4 , and 8 after both picrotoxin injections. Three rats also received vehicle injection through the same cannula, 7 days before picrotoxin treatment. Since the saline injection did not have a significant effect on theta power in either of these rats $(t[12]=-0.411, p=0.69$; $t[14]=-0.449, p=0.66, t[13]=-0.493, p=0.63$; $t$-test 2 -sided $)$, for further analysis, this period was pooled with other recordings of the pre-injection baseline period. 
A

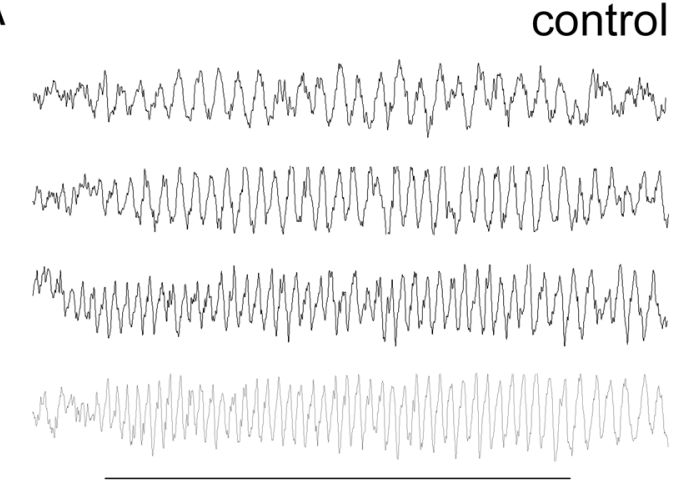

B

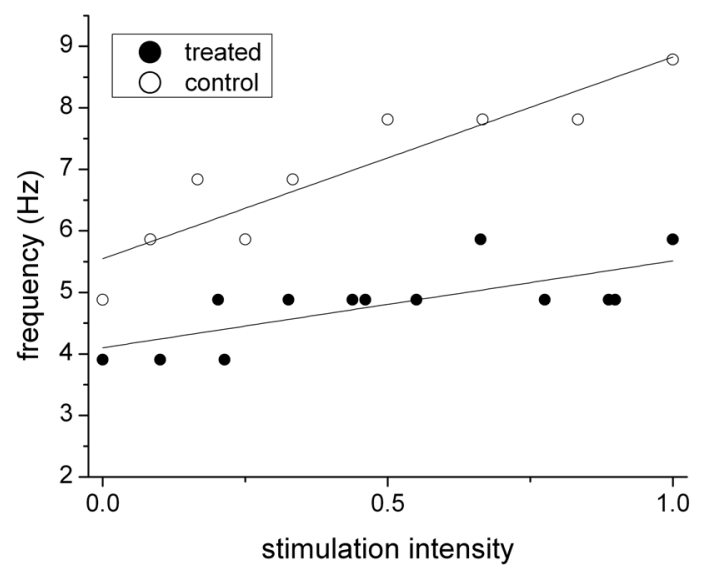

FIGURE 3 | Delayed attenuation of the hippocampal theta response to brainstem activation, $\mathbf{9 6} \mathrm{h}$ after picrotoxin injection in the BLA. (A) Sample traces of hippocampal EEG of urethane anesthetized rats during RPO stimulation (see markers) at increasing intensities, 4 days after picrotoxin injection in the BLA (right) or in the adjacent area (anatomical control, left). Note strong modulation of theta frequency in the control rat (frequency increase from 4.5 to $8.5 \mathrm{~Hz}$ ) and

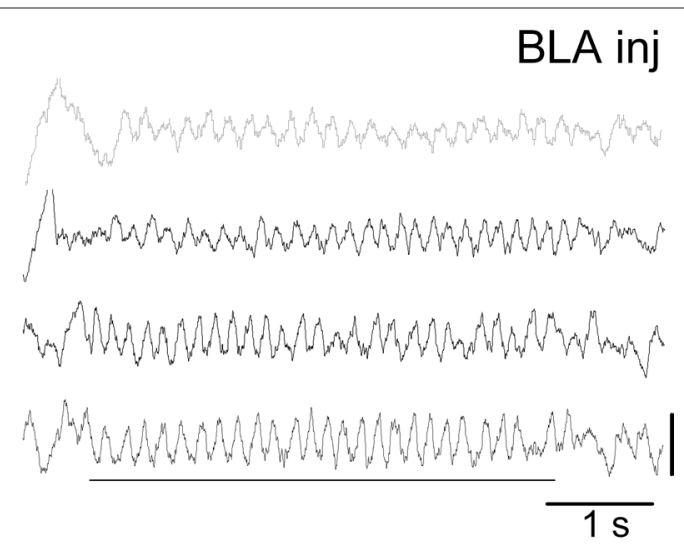

C

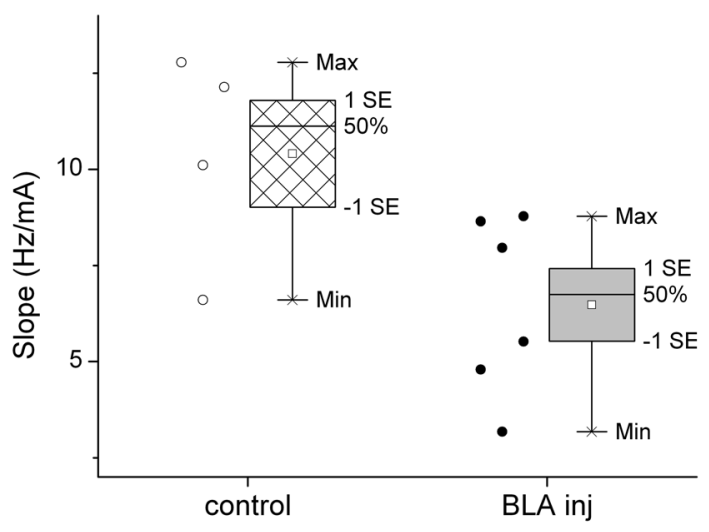

attenuated response in the BLA injected rat (frequency between 4.0 and $5.5 \mathrm{~Hz}$ ). (B) Linear relationship between stimulus intensity and theta frequency in a control and BLA injected rat (R2 $=0.85$ and 0.54 , respectively). (C) Comparison of the theta frequency vs. stimulus intensity relationship in the group of four control and six BLA injected rats (individual experiments are shown by circles on left, group statistics by box-and-whisker plots on right).
The recordings lasted for at least $4 \mathrm{~h}$ on each recording day. On visual inspection, no gross alterations were seen in the hippocampal EEG of any of the seven rats, post-injection (Figure 4A). The rats spent most of the first 30-50 min of recordings awake, exhibiting normal hippocampal theta rhythm during active "exploratory" behaviors, e.g., sniffing, rearing, walking, etc, and non-theta activity during consummatory behaviors such as grooming, eating, or quiet waking. During the 2nd and 3rd hours of the recording sessions, hippocampal EEG showed cycles of normal sleep-wake patterns with well defined large delta waves and sleep spindles during slow wave sleep, theta rhythm during REM sleep, and spindles on theta background during transitional sleep. Also, no evidence of seizure activity was found in any rats at any time point.

In spectral analysis, pre- and post-injection observations for BLA-treated rats were used for statistical comparisons. Changes in power were calculated as band averages $(5-10 \mathrm{~Hz}$ for theta and 30-50 Hz for gamma) over long continuous recordings and then separately for theta-intensive segments. For theta rhythm, changes in peak power were also analyzed (Table 1). First, 3 h continuous recordings were used which were not focused on specific behaviors, but instead were based on observation of EEG oscillations in the context of regular activity during the light period of the day. For each rat, these recordings were sorted between pre- and post-injection data and the mean theta and gamma powers over the 1st hour (i.e., mostly awake) and hours 2-3 (quiet waking and sleep) of each recording session were calculated and expressed as a ratio relative to average pre-injection baseline. The effects of the injection on the strength of oscillations differed between CA1 and DG (Figures 4C,D). Thus, in the CA1, there was a general tendency of increase in both theta and gamma powers whereas DG theta and gamma either decreased or did not change. The changes of theta and gamma powers in CA1 and DG were analyzed separately for the 1st hour and for hours 2-3 using two-way ANOVA with time (three recordings before and four recordings after injection) and area (CA1 and DG) as main factors. The different tendencies in CA1 and DG were confirmed by significant time-area interactions for theta and gamma in hours $2-3$ (theta hour $1: F[6,89]=2.108$, $p=0.06$; theta hour 2-3: $F[6,89]=2.208, p=0.049$; gamma hour 1 : $F[6,87]=1.295, p=0.27$; gamma hour $2-3: F[6,89]=2.977, p=0.01)$ and by significant main effect of area (theta hour $1: F[1,89]=7.819$, 
A

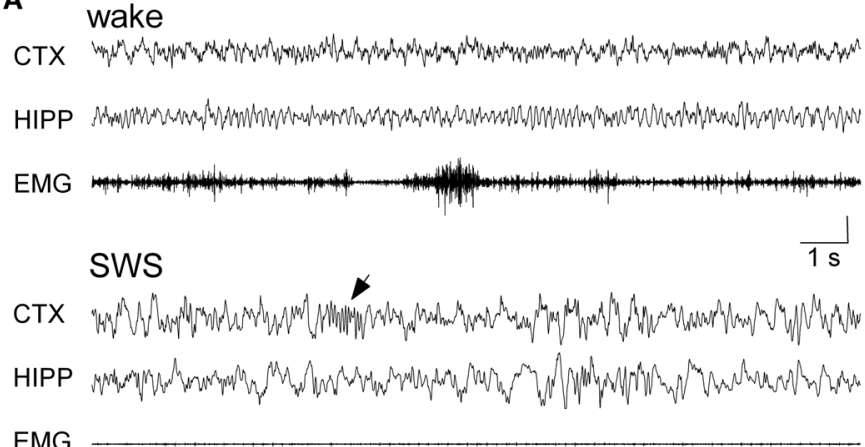

\section{REM}

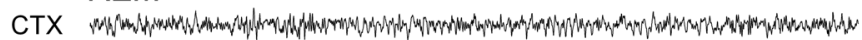

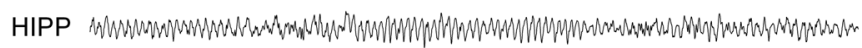

EMG

B

REM

I-CA1 WWMWMWMWI

r-CA1 WWWWWWWWVI

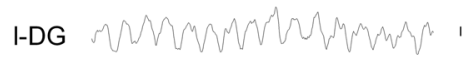

r-DG WWWAMWWN' wake

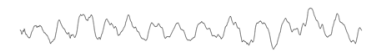

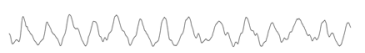

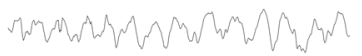

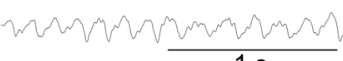

C
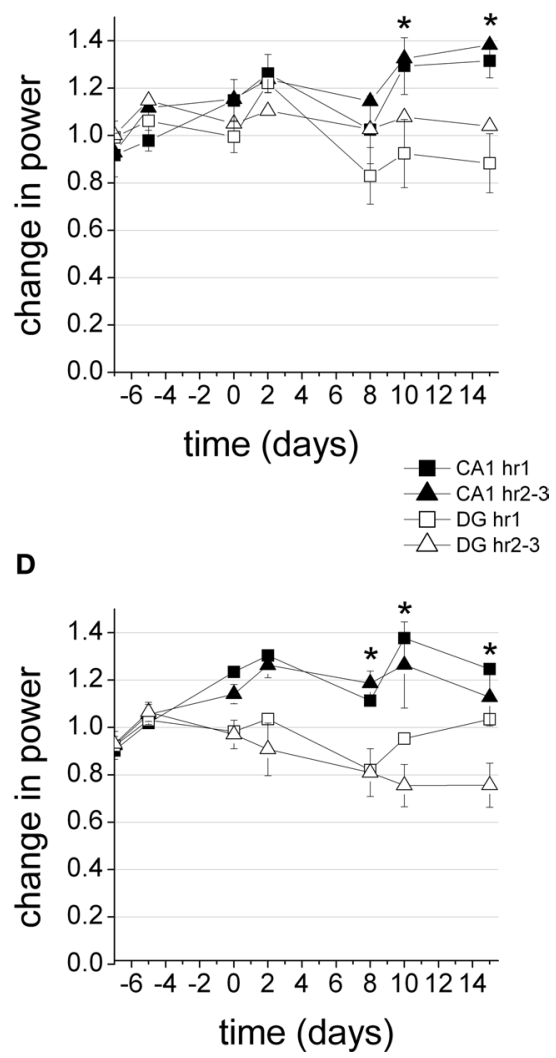

FIGURE 4 | Hippocampal oscillations after picrotoxin injection in BLA in freely behaving rats. (A) Specimen recordings of frontal cortical (CTX) and hippocampal (HIPP) EEG and muscle activity (EMG) in different sleep-wake states, 8 day after injection (SWS - slow wave sleep, arrow: sleep spindle). (B) Field potentials at the major theta dipoles. Note phase ( 180 deg) and amplitude (DG > CA1) difference (EEG calibration $1 \mathrm{mV}$ ). (C,D) Average theta (C) and gamma (D) power during the 1st hour and hours 2-3 of seven recording sessions, prior to BLA treatment (days $-7,-5,0$ ), as well as 2 and 8 days after the first (days 2, 8) and second (days 10, 15) injection. For each rat, the spectral power in the theta $(5-10 \mathrm{~Hz})$ and gamma $(30-50 \mathrm{~Hz})$ bands was normalized and expressed as a ratio relative to average baseline power during the last three pre-injection recording days. Error bars and asterisks to indicate significant CA1 and DG difference were added for "hour 2-3" recordings, in which time-area interactions were significant at $p=0.05$ level.

Table 1 | Summary of changes in theta and gamma power after picrotoxin injections in baso-lateral amygdala.

\begin{tabular}{|c|c|c|c|c|c|}
\hline & \multicolumn{2}{|c|}{ Theta } & \multicolumn{2}{|c|}{ Gamma } & \multirow{2}{*}{$\begin{array}{l}\text { Significant in } \\
\text { two-way ANOVA }\end{array}$} \\
\hline & CA1 & DG & CA1 & DG & \\
\hline Ave pwr-1sth & $1.30+0.17$ & $0.89+0.19$ & $1.40+0.27^{*}$ & $0.97+0.21$ & T,G: area, T: time \\
\hline NOV (freq band) & $1.60+0.50$ & $0.92+0.19$ & $1.40+0.30$ & $1.33+0.26$ & $\mathrm{~T}$ : area \\
\hline NOV (peak pwr) & $1.26+0.20$ & $0.82+0.05^{*}$ & & & \\
\hline REM (freq band) & $1.67+0.45$ & $0.94+0.20$ & $1.31+1.10$ & $1.29+0.42$ & $\mathrm{~T}$ : area \\
\hline REM (peak pwr) & $1.44+0.14^{*}$ & $1.05+0.04$ & & & \\
\hline
\end{tabular}

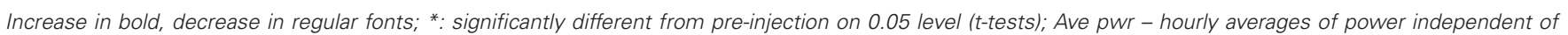

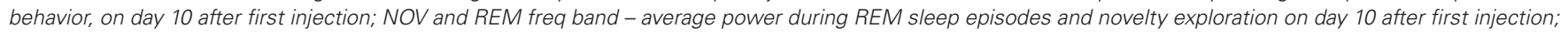

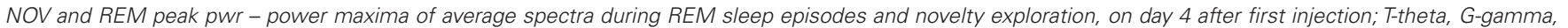
area: main effect of area (CA1 vs. DG), time: days pre-post-injection (recordings after first injection, see text).

$p<0.01$; theta hour 2-3: $F[1,89]=10.470, p<0.01$; gamma hour $1: F[1,87]=13.434, p<0.01$; gamma hour $2-3: F[1,89]=27.333$, $p<0.01)$. On the other hand, the effect of time was not significant for most comparisons (theta hour 1: $F[6,89]=2.377, p=0.04$; theta hour 2-3: $F[6,89]=1.304, p=0.26$; gamma hour $1: F[6,87]=1.999$, $p=0.07$; gamma hour 2-3: $F[6,89]=0.972, p=0.45)$. A similar 
two-way ANOVA did not reveal significant effect of side (left vs. right) in either comparison (neither main effects of side nor timeside interactions were significant).

\section{EFFECT OF PICROTOXIN INJECTION IN BLA IN HIPPOCAMPAL EEG IN THETA-INTENSIVE BEHAVIORS}

Next, we examined the hippocampal oscillations during specific behaviors with theta prominence, i.e., REM sleep and exploration of NOV (Figure 5). Recordings of 90-200 s in duration were obtained pre-injection and $96 \mathrm{~h}$ following injection. Again, the differences between hemispheres were not significant for either the REM or novelty recordings $(t[36]=-1.32, p=0.098$, Paired 2 sided $t$-test). This was also true of the control rats $(t[15]=-0.63$, $p=0.54)$. Since the injection did not have a unilateral effect, distinctions between brain hemispheres were removed from further analysis.

Theta and gamma oscillations in REM and NOV recordings were analyzed using average power in the corresponding frequency bands (Figure 5A), as well as the theta peak of the average spectra (Figures 5B,C). For band power, two-way ANOVA with area (CA1 vs. DG) and time (pre- vs. post-injection) as main factors, showed significant CA1-DG difference $(F[1,47]=5.113, p=0.029)$. Interaction and time effects were however below the level of significance, most likely due to relatively large variations between experiments, even though increases in CA1 theta power reached above 50\%, on average (Figure 5A; REM: $1.78+0.67$; NOV: $1.59+0.51$ ). There were no changes in DG during REM $(1.14+0.09)$ or novelty exploration $(1.02+0.05)$ and the changes in gamma band activity were not significant in either area in any behavior (Figure 5A). These values remained similar after additional picrotoxin injection on the other side (Table 1 ).

The changes in the theta peak of the average spectra also differed between CA1 and DG and were analyzed separately. Comparison of pre- and post-injection recordings in BLA injected rats showed highly uneven but significant increases in both CA1 $(1.44 \pm 0.13$, $t[6]=2.45, p=0.007$, paired $t$-test $)$ and DG $(1.05 \pm 0.003$, $t[6]=2.015, p=0.008)$ theta peak during REM sleep and a significant decrease in theta power associated with novelty in the DG $(0.82 \pm 0.023, t[5]=2.57, p=0.046)$ but not in the CA1 region $(1.26 \pm 0.27, t[6]=2.44, p=0.24)$. There were no significant changes in peak theta power in the control rats (Figure 5B). When compared with controls, the CA1 theta increase in BLA injected rats during $\operatorname{REM}(t[8]=2.306, p=0.036)$ and the decrease in DG during exploration proved significantly different $(t[7]=2.36, p=0.018)$, indicating a suppression of NOV-related DG-theta and enhancement of REM-related CA1-theta 4 days after picrotoxin injection in the BLA. All signals were highly coherent (Kocsis et al., 1999) and the peak coherence did not change after the injection. The relative phase between ipsilateral CA1 and DG recordings and between contralateral CA1 recordings were measured in each recording and were found unchanged over time in any of the rats included in the analysis (CA1-DG/REM: $162+4^{\circ}$ to $166+5^{\circ}, p=0.68$; CA1-DG/ NOV: $163+6^{\circ}$ to $162+7^{\circ}, p=0.166$; CA1-CA1/REM: $16+8^{\circ}$ to $5+1,11+6^{\circ}, p=0.71 ; \mathrm{CA} 1-\mathrm{CA} 1 / \mathrm{NOV}: 15+13^{\circ}$ to $\left.8+4^{\circ}, p=0.39\right)$ indicating that the relative phase between the two theta dipoles was not affected by BLA injection or time. These data also show that the electrode position did not change substantially during the
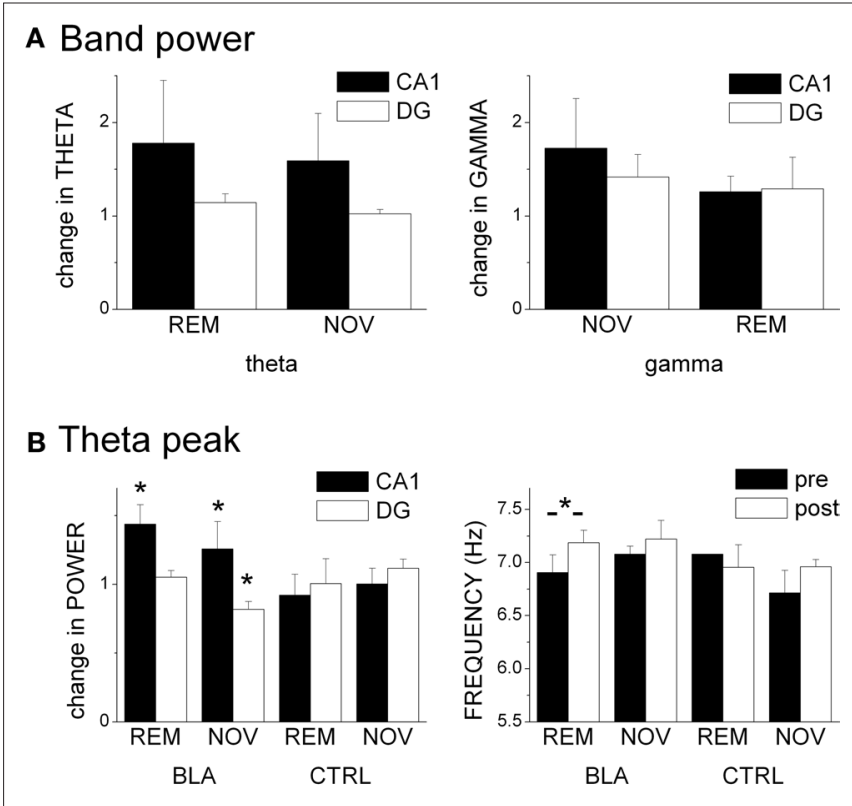

\section{Theta peak power}
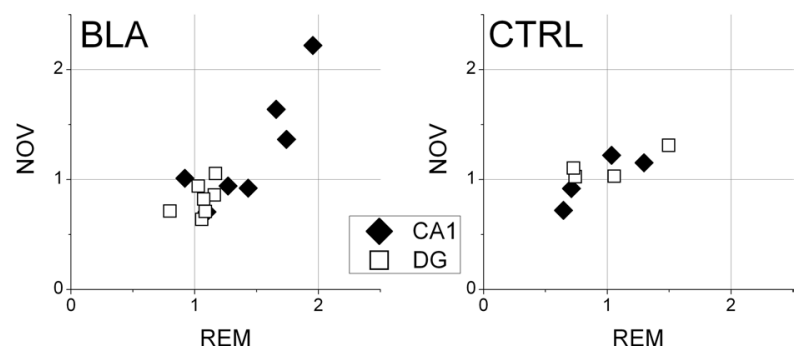

FIGURE 5 | Effect of picrotoxin injection in the BLA on hippocampal oscillations during REM sleep and novelty exploration. (A) Change in spectral power in the theta $(5-10 \mathrm{~Hz})$ and gamma $(30-50 \mathrm{~Hz})$ frequency ranges in $\mathrm{CA} 1$ and $\mathrm{DG}$ regions, expressed as ratio of average band power before and on the 4th day after BLA injection. (B) Characteristics of the theta peak of the average spectra. Theta frequency is shown in absolute values, theta power is expressed as pre- post-injection ratio. (C) Changes in theta power in REM and NOV after BLA (left) and control injections (right) of picrotoxin in individual experiments.

experiments and excludes the possibility of theta power fluctuations over time due to swelling or shrinking of the brain that might, in principle, take place postoperatively.

\section{DISCUSSION}

The results of this study indicate that sub acute disruption of interneuron signaling in the hippocampus mediated through an over-activation of the input from the amygdala, as a model of schizophrenia, results in complex alterations of oscillation patterns in the hippocampus. In experiments under urethane anesthesia we found that an initial increase in the theta rhythm response to brainstem stimulation which began immediately following picrotoxin injection in the BLA turned to a significant decrease in the sensitivity of the theta generating network to this same stimulus after 4 days, the time when changes in interneurons resembling human schizophrenia were reported to occur in this model (Berretta and Benes, 
2006). The decrease in sensitivity was expressed by a diminished response of theta frequency to increasing brainstem stimulation. In chronic rats, the most consistent findings were disparities between the BLA injection induced alterations at the two major theta dipoles, i.e., in the CA1 pyramidal layer and at the hippocampal fissure. Significant differences between the changes observed in the two layers were found in theta and gamma power averaged over different behaviors in the home cage, and specifically during thetaintensive behaviors of REM sleep and novelty exploration. Pairwise post hoc comparison showed significant CA1 vs. DG differences in post-injection recordings. However, absolute changes in theta power in pre- vs. post-injection comparisons were only significant for the $44 \%$ increase in peak theta power in the CA1 region and for an $18 \%$ decrease in peak theta power of the deep theta generator recorded in the DG. In total theta band power, a 25-70\% increase in CA1 was seen consistently contrasted with a $10-15 \%$ decrease or no change in DG (Table 1) but the increase was not significant, potentially due to interindividual variability in the delayed reaction to the intervention. The results indicate that pathologic reorganization of interneuron circuits i.e., decrease, increase and no change, respectively, in the number of GABAergic interneurons and/or terminals expressing parvalbumin, cholecystokinin, and calretinin, as reported for this model (Berretta et al., 2004) does not lead to destruction of the oscillatory circuit but changes the normal balance of rhythmic activity in its various compartments. As theta field in the CA1 is generated by IPSPs, as opposed to deep layers where it is driven by EPSPs (Buzsaki et al., 1986; Brankack et al., 1993), these data indicate that changes in hippocampal interneurons are the primary origin of the altered activity pattern.

\section{THE EFFECT OF COMPLEX REORGANIZATION OF INTERNEURON NETWORKS INDUCED BY BLA ACTIVATION ON HIPPOCAMPAL OSCILLATIONS}

Interneurons can in principle contribute to the generation of EEG rhythms in two different ways, i.e., acting either on the "current generator" or on the "rhythm generator" (Buzsaki, 2002). In the first case, they generate IPSPs on pyramidal cells which directly contribute to the magnitude of the recorded field under certain circumstances; i.e., if they fire rhythmically and in synchrony and if they target a large number of pyramidal cells simultaneously and in a layer-specific manner. In the second, different types of interneurons are part of the "rhythm generator" which is responsible for the emergence and control of the oscillatory pattern and frequency. This depends on their unique membrane properties, network connections with other GABAergic cells, and/or reciprocal connections with excitatory unit populations (Whittington and Traub, 2003; Gloveli et al., 2005).

\section{The role of $\mathrm{PV}+$ interneurons}

Earlier histological analysis of the hippocampus in this model (Berretta et al., 2004) documented region-specific decrease in the numbers of PV + cells (smallest in CA1) and PV+ terminals (largest in CA1). Of all the different interneuron classes, PV+ basket cells have the largest "current generator" effect as they provide most of the synaptic input on the pyramidal cells soma and proximal dendrites. Synchronized, rhythmic IPSPs by this group are the major source of the theta dipole (a current source, see Buzsaki et al., 1986;
Brankack et al., 1993; Bragin et al., 1995) in the CA1 pyramidal layer, recorded in our experiments. These interneurons are also critical components of the hippocampal "rhythm generator". They exhibit the appropriate fast, non-accommodating pattern of firing, and are highly interconnected between each other by fast GABA-A signaling as well as gap junctions. $\mathrm{PV}+$ basket cells also receive rhythmic input from the theta pacemaker in the medial septum (Freund and Antal, 1988), and their distal dendrites are also reached by rhythmic perforant path input (Kiss et al., 1996). Nevertheless, in our study, even if the increase was not always significant, a possible decrease in CA1 theta power could confidently be excluded, contrary to what could be expected after loss of PV+ interneurons (cells and/or terminals).

\section{Changes of basket cell terminals}

Several days after amygdalar activation, Berretta et al. (2004) found a significant increase in the total number of GAD65+ puncta in all regions of the hippocampus, the largest in the CA1 region. This could explain the enhanced amplitude of theta and gamma fields in the CA1, found in this study, provided that the additional GABA input effectively transmitted rhythmic activity. The GAD65+ increase was mostly due to increase in the number of $\mathrm{CCK}+$ puncta which compensated for the significant loss of PV+ terminals in the same layers. Such a change in the proportion of the two types of basket cells may be unfavorable for oscillations as for driving fast rhythmic IPSPs, both the firing rate and pattern of $\mathrm{CCK}+$ basket cells are inferior to their PV+ counterparts. However, $\mathrm{CCK}+$ interneurons do fire rhythmically and thus contribute to the field at a somewhat different phase of the theta cycle (Klausberger et al., 2005). It was also shown that hippocampal oscillations can be manipulated by compounds acting on receptors expressed by CCK+ but not by PV+ interneurons (Siok et al., 2006; Hajos et al., 2008). On the other hand, a decrease in PV+ puncta may reflect a compensatory decrease in PV expression rather than a loss of GABA terminals, consistent with the finding that the decrease in PV+ terminals was not accompanied by an equal loss of PV+ cell somata in the CA1 layer (Berretta et al., 2004). Indeed, a decrease in PV expression was proposed (Lewis et al., 2005) as a compensatory mechanism in schizophrenia to balance the deficit in GABAergic input to pyramidal cells. By buffering presynaptic $\mathrm{Ca}++$ transients, PV limits GABA release during repetitive firing. Therefore, PV downregulation may lead to higher residual presynaptic $\mathrm{CA}++$ levels and subsequent elevation of GABA release and increased IPSP amplitude. In fact, increased GABA release and enhanced gamma oscillations have been shown in PV-knockout mice (Vreugdenhil et al., 2003). Furthermore, it has been shown that the PV+ type of hippocampal interneurons are specifically responsive to changes in CCK levels (Foldy et al., 2007). CCK receptors are mostly expressed by pyramidal cells but they are also found in interneurons. Thus, increase in CCK release in the network can selectively activate PV+ neurons and serve as an additional compensatory mechanism to prevent deterioration of oscillatory function.

\section{Other interneurons}

The contribution of the second type of $\mathrm{PV}+$ perisomatic interneurons, the chandelier cells, to the field is less obvious (Szabadics et al., 2006), but they have an enormous potential to synchronize large 
populations of pyramidal cells (Li et al., 1992). Although we have not seen gross failures of the oscillatory circuits in this model, fine alterations in the effectiveness of synchronization could contribute to some of the observed changes in theta and gamma amplitude and theta frequency; for example to the more prominent increase in peak theta amplitude as compared with theta band power. Similarly, dendritic-targeting PV+ cells have smaller effects as current generators but may play an important role as rhythm generators. The termination of bistratified cells is too widely dispersed to generate detectable field potentials and the oriens-lacunosum moleculare (O-LM) cells terminate at the location of the largest theta dipole generated by massive current sinks due to rhythmic perforant path EPSPs. Nevertheless, O-LM cell IPSPs, arriving at the positive peak of the local field, can significantly shape and enhance the EPSP-generated oscillation. O-LM cells possess the membrane machinery to intrinsically generate oscillations and they participate in the pyramidal cell-interneuron reciprocal loop (Gillies et al., 2002; Orban et al., 2006; Tort et al., 2007) both in the CA1 and CA3 regions. Since the changes in interneurons were larger in the CA 3 than in CA1 (Berretta et al., 2004), increased CA1 theta in this model may also reflect the absence of interference between these two sources. The possible effect of the region-specific loss of $\mathrm{CR}+$ and $\mathrm{CB}+$ interneurons on oscillations is also due to their role in the rhythm generator. Similar to PV+ basket cells, CR+ interneurons also form a highly connected syncytium but these only affect pyramidal cells through other, non-PV+ interneurons (Gulyas et al., 1996).

\section{THE RELEVANCE OF MORPHOLOGICAL AND ELECTROPHYSIOLOGICAL FINDINGS INDUCED BY BLA DISINHIBITION FOR SCHIZOPHRENIA, COMPARISON WITH OTHER MODELS \\ $P V+$ cell deficiency is common for most models of schizophrenia}

Region-specific loss of PV+ neurons has been reported in postmortem human schizophrenic brains (Zhang and Reynolds, 2002). It is remarkable that, similar to behavioral assays, e.g., dopaminedependent hyperactivity, sensory gating, etc. (Carpenter and Koenig, 2008), this feature also appears in most animal models, even if they are based on widely different approaches, including manipulations in adult animals (Keilhoff et al., 2004; Rujescu et al., 2006), on neonates (Tseng et al., 2008; Francois et al., 2009), or in embryonic life (Penschuck et al., 2006; Lodge et al., 2009). The technique used by Berretta and Benes (2006) to generate their "partial" model did not specifically target PV+ neuron development or function; instead, their goal was to dissect out one distant source of afferent activity to the hippocampus that may be involved in the pathomechanism of schizophrenia. Nevertheless, PV+ deficiency was one important component of the resulting interneuron constellation. The well-known role of PV+ interneurons in rhythm generation suggests that the disturbances in cortical oscillations in schizophrenia (Spencer et al., 2008b) are a consequence of this characteristic morphology - a viable hypothesis, still lacking direct proof, however. PV+ cell loss induced by such a diversity of techniques most likely appears on different network backgrounds, and the functional consequences may differ between models. Specifically, in the model of Berretta et al. (2004), PV+ neuron deficit (cell number and terminals) did not lead to decrease in oscillation power indicating that knowing this feature alone is not sufficient to predict network activity.

\section{Network oscillations are altered in models of schizophrenia}

In this study we analyzed state-dependent rhythms, rather than task-related evoked or induced oscillations. It was shown in human schizophrenics that gamma power increased in REM sleep, in contrast to reduced gamma oscillations in cognitive tasks (Tekell et al., 2005). Slow oscillations were also enhanced in schizophrenics at rest, but functionally impaired in cognitive tasks (Bates et al., 2009). Discrepancies between background and evoked oscillations in the theta and gamma ranges were also shown recently in rodents after acute ketamine administration (Lazarewicz et al., 2010). The cellular mechanism of such discrepancies and how they relate to $\mathrm{PV}+$ neuron deficits are not known; to our knowledge there is no data indicating that PV+ interneurons would be selectively involved in task-related oscillations but not in state-dependent rhythms. One possibility is that CCK+ neuron activity which is under control of various neuromodulators (Freund, 2003) may specifically enhance PV+ network-dependent oscillations (Foldy et al., 2007) in certain states but not in others. Future studies will have to evaluate the relationship between stimulus evoked and induced rhythms and background oscillations in the BLA activation model.

When comparing animal models, it is also important to differentiate between acute and chronic designs and consider the timing of different features in relation to the course of schizophrenia. Since this model uses adult animals, it obviously ignores genetic and neurodevelopmental aspects. It was hypothesized however, that pharmacological disinhibition of BLA in the rat may reconstruct a sequence of events that is induced in schizophrenics at a certain age as a consequence of abnormal GABA transmission in the amygdala. Berretta et al. (2004) also argued that the reorganization of interneuron circuits in the hippocampus observed 4 days after treatment might be an intermediate stage of a chronic response of GABA cells to amygdalar activation. This is supported by a change in the number of GAD65+ terminals from a decrease $2 \mathrm{~h}$ after BLA injection (Berretta et al., 2001) to an increase $96 \mathrm{~h}$ later (Berretta et al., 2004), and is consistent with the present observation of the difference in theta generation elicited by brainstem stimulation immediately after the injection and 4 days later. Furthermore, some of the more severe functional consequences were reported to occur 15 days after treatment (Gisabella et al., 2009). The increase in GAD65+ terminals, just as the decrease of PV expression in terminals, may thus represent an adaptive mechanism manifested at this early stage which might become unsustainable or inadequate as the disease progresses (Lewis et al., 2005).

\section{Amygdalar modulation of hippocampal function in schizophrenia}

Patients with schizophrenia demonstrate significant impairments in processing emotions (for review see Shayegan and Stahl, 2005), which is linked with documented morphological changes and malfunctioning of the amygdala (Keshavan et al., 2002; Tomasino et al., 2010). The amygdala has a strong effect on hippocampal processing of emotional information and the manipulation of this connection resulted in complex changes in multiple interconnected populations of hippocampal interneurons (Berretta et al., 2004). It was demonstrated in particular that CCK+ terminals are regulated by other interneurons and this regulation was affected by the amygdala. GABA blockade in the amygdala changed the 
balance from local within-sector control by $\mathrm{CR}+$ interneurons to long-range correlation of $\mathrm{CCK}+$ terminals in $\mathrm{CA} 1-\mathrm{CA} 3$ with $\mathrm{PB}+$, $\mathrm{CR}+$ and $\mathrm{PV}+$ interneurons in other sectors. The relatively high density of CCK+ interneurons in the amygdala and hippocampus and their unique set of receptors for neuromodulators indicate that they are involved in emotional and motivational processing (Freund, 2003). Cannabinoid CB1, alpha-7 nicotonic, 5-HT3, GABAB receptors are among those expressed by CCK+ but not by $\mathrm{PV}+$ neurons. Based on a variety of contrasting features, Freund (2003) proposed that these two types of basket cells are engaged in orthogonally different functions, the first representing a syncytium of fine-tuning devices conveying information related to emotion

\section{REFERENCES}

Basar-Eroglu, C., Brand, A., Hildebrandt, H., Karolina Kedzior, K., Mathes, B., and Schmiedt, C. (2007). Working memory related gamma oscillations in schizophrenia patients. Int. J. Psychophysiol. 64, 39-45.

Basar-Eroglu, C., Schmiedt-Fehr, C., Marbach, S., Brand, A., and Mathes, B. (2008). Altered oscillatory alpha and theta networks in schizophrenia. Brain Res. 1235, 143-152.

Bates, A. T., Kiehl, K. A., Laurens, K. R., and Liddle, P.F. (2009). Low-frequency EEG oscillations associated with information processing in schizophrenia. Schizophr. Res. 115, 222-230.

Beasley, C. L., and Reynolds, G. P. (1997). Parvalbumin-immunoreactive neurons are reduced in the prefrontal cortex of schizophrenics. Schizophr. Res. 24, 349-355.

Berretta, S., and Benes, F. M. (2006). A rat model for neural circuitry abnormalities in schizophrenia. Nat. Protoc. 1, 833-839.

Berretta, S., Lange, N., Bhattacharyya, S., Sebro, R., Garces, J., and Benes, F. M. (2004). Long-term effects of amygdala GABA receptor blockade on specific subpopulations of hippocampal interneurons. Hippocampus 14, 876-894.

Berretta, S., Munno, D. W., and Benes, F. M. (2001). Amygdalar activation alters the hippocampal GABA system: "partial" modelling for postmortem changes in schizophrenia. J. Comp. Neurol. 431, 129-138.

Bragin, A., Jando, G., Nadasdy, Z., Hetke, J., Wise, K., and Buzsaki, G. (1995). Gamma (40-100 Hz) oscillation in the hippocampus of the behaving rat. $J$. Neurosci. 15, 47-60.

Brankack, J., Stewart, M., and Fox, S. E. (1993). Current source density analysis of the hippocampal theta rhythm: associated sustained potentials and candidate synaptic generators. Brain Res. 615, 310-327.

Buzsaki, G. (2002). Theta oscillations in the hippocampus. Neuron 33, 325-340.
Buzsaki, G., Czopf, J., Kondakor, I., and Kellényi, L. (1986). Laminar distribution of hippocampal rhythmic slow activity (RSA) in the behaving rat: current source density analysis. Brain Res. 365, 125-137.

Buzsaki, G., Horvath,Z., Urioste, R., Hetke, J., and Wise, K. (1992). High frequency network oscillation in the hippocampus. Science 256, 1025-1027.

Carpenter, W. T., and Koenig, J. I. (2008). The evolution of drug development in schizophrenia: past issues and future opportunities. Neuropsychopharmacology 33, 2061-2079.

Foldy, C., Lee, S. Y., Szabadics, J., Neu, A., and Soltesz, I. (2007). Cell typespecific gating of perisomatic inhibition by cholecystokinin. Nat. Neurosci. $10,1128-1130$.

Ford, J. M., Mathalon, D. H., Whitfield, S., Faustman, W. O., and Roth, W. T. (2002). Reduced communication between frontal and temporal lobes during talking in schizophrenia. Biol. Psychiatry 51, 485-492.

Francois, J., Ferrandon, A., Koning, E., Angst, M. J., Sandner, G., and Nehlig, A. (2009). Selective reorganization of GABAergic transmission in neonatal ventral hippocampal-lesioned rats. Int. J. Neuropsychopharmacol. 12, 1097-1110.

Freund, T.F. (2003). Interneuron diversity series: Rhythm and mood in perisomatic inhibition. Trends Neurosci. 26, 489-495.

Freund, T.F., and Antal, M. (1988). GABAcontaining neurons in the septum control inhibitory interneurons in the hippocampus. Nature 336, 170-173.

Gallinat, J., Winterer, G., Herrmann, C. S., and Senkowski, D. (2004). Reduced oscillatory gamma-band responses in unmedicated schizophrenic patients indicate impaired frontal network processing. Clin. Neurophysiol. 115, 1863-1874.

Gillies, M. J., Traub, R. D., LeBleau, F. E. N., Davies, C. H., Gloveli, T., Buhl, E. H., and Whittington, M. A. (2002). A model of atropine-resistant theta

and general state, the second operating the machinery of principal cells ensembles. Changing the balance between these systems may contribute to pathologic emotional processing in schizophrenia. Altered oscillations may be involved as an important mechanism as some of these neuromodulators were shown to have strong effect on hippocampal rhythms, e.g., CB1 receptors interfering with rhythmic synchronization (Robbe et al., 2006; Hajos et al., 2008), alpha-7 nicotininc receptors supporting rhythmic activity (Siok et al., 2006).

\section{ACKNOWLEDGMENT}

This study was sponsored by NIMH (Grant No. MH083199).

oscillations in rat hippocampal area CA1. J. Physiol. (Lond.) 543 779-793.

Gisabella, B., Cunningham, M. G., Bolshakov, V. Y., and Benes, F. M. (2009). Amygdala-dependent regulation of electrical properties of hippocampal interneurons in a model of schizophrenia. Biol. Psychiatry 65, 464-472.

Gloveli, T., Dugladze, T., Saha, S., Monyer, H., Heinemann, U., Traub, R. D., Whittington, M. A., and Buhl, E. H (2005). Differential involvement of oriens/pyramidale interneurones in hippocampal network oscillations in vitro. J. Physiol. 562, 131-147.

Gonzalez-Burgos, G., and Lewis, D. A. (2008). GABA neurons and the mechanisms of network oscillations: implications for understanding cortical dysfunction in schizophrenia. Schizophr. Bull. 34, 944-961.

Gulyas, A. I., Hajos, N., and Freund, T. F. (1996). Interneurons containing calretinin are specialized to control other interneurons in the rat hippocampus. J. Neurosci. 15, 3397-3411.

Hajos, M., Hoffmann, W. E., and Kocsis, B. (2008). Activation of cannabinoid-1 receptors disrupts sensory gating and neuronal oscillation: relevance to schizophrenia. Biol. Psychiatry 63, 1075-1083.

Hikida, T., Jaaro-Peled, H., Seshadri, S. Oishi, K., Hookway, C., Kong, S., Wu, D., Xue, R., Andrade, M., Tankou, S. Mori, S., Gallagher, M., Ishizuka, K. Pletnikov, M., Kida, S., and Sawa, A. (2007). Dominant-negative DISC1 transgenic mice display schizophrenia-associated phenotypes detected by measures translatable to humans. Proc. Natl. Acad. Sci. U.S.A. 104 14501-14506.

Hong, L. E., Summerfelt, A., McMahon, R., Adami, H., Francis, G., Elliott, A., Buchanan, R. W., and Thaker, G. K. (2004). Evoked gamma band synchronization and the liability for schizophrenia. Schizophr. Res. 70, 293-302.

Jaaro-Peled, H., Ayhan, Y., Pletnikov, M. V., and Sawa, A. (2010). Review of pathological hallmarks of schizophrenia: comparison of genetic models with patients and nongenetic models. Schizophr. Bull. 36, 301-313.

Keilhoff, G., Becker, A., Grecksch, G., Wolf, G., and Bernstein, H. G. (2004) Repeated application of ketamine to rats induces changes in the hippocampal expression of parvalbumin, neuronal nitric oxide synthase and cFOS similar to those found in human schizophrenia. Neuroscience 126, 591-598.

Keshavan, M. S., Dick, E., Mankowski, I., Harenski, K., Montrose, D. M., Diwadkar, V., and DeBellis, M. (2002). Decreased left amygdala and hippocampal volumes in young offspring at risk for schizophrenia. Schizophr. Res. 58, 173-183.

Kiss, J., Buzsaki, G., Morrow, J. S., Glantz, S. B., and Leranth, C. (1996). Entorhinal cortical innervation of parvalbumincontaining neurons (basket and chandelier cells) in the rat Ammon's horn. Hippocampus 6, 239-246.

Klausberger, T., and Somogyi, P. (2008). Neuronal diversity and temporal dynamics: the unity of hippocampal circuit operations. Science 321, 53-57.

Klausberger, T., Marton, L. F., O’Neill, J., Huck, J. H., Dalezios, Y., Fuentealba, P., Suen, W. Y., Papp, E., Kaneko, T., Watanabe, M., Csicsvari, J., and Somogyi, P. (2005). Complementary roles of cholecystokinin- and parvalbumin-expressing GABAergic neurons in hippocampal network oscillations. J. Neurosci. 25, 9782-9793.

Kocsis, B., Bragin, A., and Buzsaki, G. (1999). Interdependence of multiple theta generators in the hippocampus: a partial coherence analysis. J. Neurosci. $19,6200-6212$.

Kocsis, B., and Li, S. (2004). In vivo contribution of h-channels in the septal pacemaker to the generation of theta rhythm in the rat. Eur. J. Neurosci. 20, 2149-2158.

Koenig, T., Lehmann, D., Saito, N. Kuginuki, T., Kinoshita, T., and Koukkou, M. (2001). Decreased 
functional connectivity of EEG theta-frequency activity in first-episode, neuroleptic-naive patients with schizophrenia: preliminary results. Schizophr. Res. 50, 55-60.

Krishnan, G. P., Vohs, J. L., Hetrick, W. P., Carroll, C.A., Shekhar,A., Bockbrader, M. A., and O'Donnell, B. F. (2005). Steady state visual evoked potential abnormalities in schizophrenia. Clin. Neurophysiol. 116, 614-624.

Lakatos, P., Karmos, G., Mehta, A. D., Ulbert, I., and Schroeder, C. E. (2008). Entrainment of neuronal oscillations as a mechanism of attentional selection. Science 320, 110-113.

Lazarewicz, M. T., Ehrlichman, R. S., Maxwell, C. R., Gandal, M. J., Finkel, L. H., and Siegel, S. J. (2010). Ketamine modulates theta and gamma oscillations. J. Cogn. Neurosci. 22, 1452-1464.

Lewis, D. A., Hashimoto, T., and Volk, D. W. (2005). Cortical inhibitory neurons and schizophrenia. Nat. Rev. Neurosci. 6, 312-324.

Li, X. G., Somogyi, P., Tepper, J. M., and Buzsaki, G. (1992). Axonal and dendritic arborization of an intracellularly labeled chandelier cell in the CA1 region of rat hippocampus. Exp. Brain Res. 90, 519-525.

Lisman, J., and Buzsaki, G. (2008). A neural coding scheme formed by the combined function of gamma and theta oscillations. Schizophr. Bull. 34, 974-980.

Lodge, D. J., Behrens, M. M., and Grace, A. A. (2009). A loss of parvalbumincontaining interneurons is associated with diminished oscillatory activity in an animal model of schizophrenia. $J$. Neurosci. 29, 2344-2354.

Orban, G., Kiss, T., and Erdi, P. (2006). Intrinsic and synaptic mechanisms determining the timing of neuron population activity during hippocampal theta oscillation. J. Neurophysiol. 96, 2889-2904.

Penschuck, S., Flagstad, P., Didriksen, M., Leist, M., and Michael-Titus, A. T. (2006). Decrease in parvalbuminexpressingneuronsinthehippocampus and increased phencyclidine-induced locomotor activity in the rat methylazoxymethanol (MAM) model of schizophrenia. Eur. J. Neurosci. 23, 279-284.

Pikkarainen, M., Ronkko, S., Savander, V. Insausti, R., and Pitkanen, A. (1999). Projections from the lateral, basal, and accessory basal nuclei of the amygdala to the hippocampal formation in rat. J. Comp. Neurol. 403, 229-260.

Robbe, D., Montgomery, S. M., Thome, A., Rueda-Orozco, P.E., McNaughton, B. L., and Buzsáki, G. (2006). Cannabinoids reveal importance of spike timing coordination in hippocampal function. Nat. Neurosci. 9 1526-1533.

Rujescu, D., Bender, A., Keck, M. Hartmann, A. M., Ohl, F., Raeder, H., Giegling, I., Genius, J., McCarley, R. W., Moller, H. J., and Grunze, H. (2006). A pharmacological model for psychosis based on N-methyl-D-aspartate receptor hypofunction: molecular, cellular, functional and behavioral abnormalities. Biol. Psychiatry 59, 721-729.

Schmiedt, C., Brand, A., Hildebrandt, H., and Basar-Eroglu, C. (2005). Event-related theta oscillations during working memory tasks in patients with schizophrenia and healthy controls. Brain Res. Cogn. Brain Res. 25, 936-947.

Shayegan, D. K., and Stahl, S. M. (2005) Emotion processing, the amygdala, and outcome in schizophrenia. Prog. Neuropsychopharmacol. Biol. Psychiatry 29, 840-845.

Siok, C. J., Rogers, J.A., Kocsis, B., and Hajos, M. (2006). Activation of alpha-7 acetylcholine receptorsaugmentsstimulationinduced hippocampal theta oscillation. Eur. J. Neurosci. 23, 570-574.

Spencer, K. M., Salisbury, D. F., Shenton, M. E., and McCarley, R. W. (2008a). Gamma-band auditory steady-state responses are impaired in first episode psychosis. Biol. Psychiatry 64, 369-375.

Spencer, K. M., Niznikiewicz, M. A. Shenton, M. E., and McCarley, R.
W. (2008b). Sensory-evoked gamma oscillations in chronic schizophrenia. Biol. Psychiatry 63, 744-747.

Steullet, P., Cabungcal, J. H., Kulak, A., Kraftsik, R., Chen, Y., Dalton, T. P., Cuenod, M., and Do, K. Q. (2010). Redox dysregulation affects the ventral but not dorsal hippocampus: impairment of parvalbumin neurons, gamma oscillations, and related behaviors. J. Neurosci. 30, 2547-2558.

Szabadics, J., Varga, C., Molnar, G., Olah, S., Barzo, P., and Tamas, G. (2006). Excitatory effect of GABAergic axoaxonic cells in cortical microcircuits. Science 5758, 233-235.

Tekell, J. L., Hoffmann, R., Hendrickse, W., Greene, R. W., Rush, A. J., and Armitage, R. (2005). High frequency EEG activity during sleep: characteristics in schizophrenia and depression. Clin. EEG Neurosci. 36, 25-35.

Tomasino, B., Bellani, M., Perlini, C. Rambaldelli, G., Cerini, R., Isola M., Balestrieri, M., Cali, S., Versace, A., Pozzi Mucelli, R., Gasparini, A., Tansella, M., and Brambilla, P. (2010). Altered microstructure integrity of the amygdala in schizophrenia: a bimoda MRI and DWI study. Psychol. Med. $1-11$.

Tort, A. B., Rotstein, H. G., Dugladze, T. Gloveli, T., and Kopell, N. J. (2007) On the formation of gamma-coherent cell assemblies by oriens lacunosummoleculare interneurons in the hippocampus. Proc. Natl. Acad. Sci. U.S.A 104, 13490-13495.

Tseng, K. Y., Lewis, B. L., Hashimoto, T. Sesack, S. R., Kloc, M., Lewis, D. A., and O'Donnell, P. (2008). A neonatal ventral hippocampal lesion causes functional deficits in adult prefrontal cortical interneurons. J. Neurosci. 28, 12691-12699.

Vohs, J. L., Chambers, R. A., Krishnan, G. P., O'Donnell, B. F., Berg, S., and Morzorati, S. L. (2010). GABAergic modulation of the $40 \mathrm{~Hz}$ auditory steady-state response in a rat model of schizophrenia. Int. J. Neuropsychopharmacol. 13, 487-497.
Vreugdenhil, M., Jefferys, J. G., Celio, M. R., and Schwaller, B. (2003). Parvalbumin-deficiency facilitates repetitive IPSCs and gamma oscillations in the hippocampus. $J$ Neurophysiol. 89, 1414-1422.

Whittington, M. A., and Traub, R. D. (2003). Interneuron diversity series: inhibitory interneurons and network oscillations in vitro. Trends Neurosci. 26, 676-682.

Williams, L. M., Whitford, T. J., Nagy, M., Flynn, G., Harris, A. W., Silverstein, S. M., and Gordon, E. (2009). Emotionelicited gamma synchrony in patients with first-episode schizophrenia: a neural correlate of social cognition outcomes. J. Psychiatry Neurosci. 34, 303-313.

Zhang, Z. J., and Reynolds, G. P. (2002). A selective decrease in the relative density of parvalbumin-immunoreactive neurons in the hippocampus in schizophrenia. Schizophr. Res. 55, 1-10.

Conflict of Interest Statement: The authors declare that the research was conducted in the absence of any commercial or financial relationships that could be construed as a potential conflict of interest.

Received: 27 May 2010; paper pending published: 09 August 2010; accepted: 17 August 2010; published online: 01 September 2010.

Citation: Lanre-Amos $T$ and Kocsis $B$ (2010) Hippocampal oscillations in the rodent model of schizophrenia induced by amygdala GABA receptor blockade. Front. Psychiatry 1:132. doi:10.3389/ fpsyt.2010.00132

This article was submitted to Frontiers in Schizophrenia, a specialty of Frontiers in Psychiatry.

Copyright (C) 2010 Lanre-Amos and Kocsis. This is an open-access article subject to an exclusive license agreement between the authors and the Frontiers Research Foundation, which permits unrestricted use, distribution, and reproduction in any medium, provided the original authors and source are credited. 